\title{
Using Feature Extraction for Human Footprints Recognition
}

\author{
Khamael Abbas Al-Dulaimi \\ Department of Computer Science ,College of Science, University of AL-Nahrain. \\ Iraq, Baghdad, Al-Jaderyia
}

\begin{abstract}
In this paper, a robust technique for identifying and recognize human-footprint images is presented. There are many methods for footprint Recognition, but most of them require segmentation or connected component analysis.. The Recognition process utilizes the determinant value that produces the features for the human-footprint. Image's determinants values are computed, by divided image into blocks then deigned Threshold (T) to extract feature. The least square criterion is then utilized to determine the similarity between the existed (in Database file) footprints with a new query footprint's images.
\end{abstract}

\section{Keywords}

footprints, Determinant value, Euclidean Distance, extract features.

\section{INTRODUCTION}

A lot of automated biometrics based identification and verification systems have been developed [1]. The biometrics features derived from fingerprints, faces [2], irises, retinas, a speaker's voice, and perhaps a variety of other characteristics. The systems are now used in a wide range of environments, such as law enforcement, social welfare, banking, and various security applications. Some systems for personal identification use fundamental biometric features derived from fingerprints and irises. To acquire the feature, subjects must input their biometrics to a sensor. Signatures [3] and speakers' voices [4] are also features useful for verification; however, obtaining these features requires the subjects' cooperation. The main problem in automatic personal identification is how to verify the sampled feature against the registered feature with high reliability. Practical methods for automated biometrics based identification have not yet been developed for use with unconstrained subjects. Problem in automatic personal identification is how to verify the sampled feature against the registered feature with high reliability. Practical methods for automated biometrics based identification have not yet been developed for use with unconstrained subjects.

Person identification using footprint can be carried out by using any one of the two important features namely static [17] and dynamic [18]. Static feature requires stand-up posture at fixed position every time from the subject, whereas the dynamic feature deals with the walking behavior. The constraint on user posture can be released when dynamic feature is taken into account. Many Footprint based recognition techniques are being developed in recent years. They make use of several features such as normalized Static footprint [18] based on Euclidean distance, so geometric information of the footprint prior to normalization into an evaluation function for personal recognition decision is included. In this paper, we propose a footprint-based personal recognition method and test its reliability [3].

\section{RELATED WORK AND CONTRIBUTION}

Recognition of Footprints has obtained increasing attentions in recent years. Most studies were based on extracting some recognizable features since they are more robust than the features of the time domain. Literatures states there are different recognizable features are adopted or suggested. In the following sections we describe the head research lines and our contribution in the field of study:

\subsection{Related Work}

Over the last decade footprint feature extraction has been actively researched for footprint recognition. The paper provides an up-to-date review of major human footprint recognition research. In Earlier sections, an overview of footprint recognition and its applications. In later sections, literature review of the most recent footprint recognition technique is presented [3].

Footprint identification is the measurement of footprint features for recognizing the identity of a user. Footprint is universal, easy to capture and does not change much across time. Footprint biometric system does not require specialized acquisition devices. Footprint image of a left leg is captured for hundred people in different angles. No special lighting is used in this setup. The foot image is positioned and cropped according to the key points. Sequential modified Haar transform is applied to the resized footprint image to obtain Modified Haar Energy (MHE) feature. The sequential modified Haar wavelet can map integervalued signals onto integer-valued signals abandoning the property of perfect reconstruction. The MHE feature is compared with the feature vectors stored in database using Euclidean Distance [4].

In other paper comparing six conventional binarization methods for the special purpose of subsequent analysis of scanned insect footprints. Anew performance introduced criterion for performance evaluation. The six different binarization methods are selected from different methodologically categories, and the proposed performance criterion is related to the specific characteristics of insect footprints of having a very small percentage of object areas. The results indicate that a higherorder entropy binarization algorithm, such as proposed by Abutaleb, offers best results for further pattern recognition application steps for the analysis of scanned insect footprints [5]. 


\subsection{Contribution}

In this paper, a new method proposed to extract features of footprint and used these feature for recognize. This framework consists of a formal model definition and a three stages algorithm for recognition. Modified the classical way of Determinant value (that be requisite input whole the footprint into determinant computation), so that the created recognition system becomes feasible (fast and accurate) for recognizing a query footprint from huge dataset also, propose a suitable similarity measure incorporating the quantitative meaningful features (Det value) with the qualitative features to get a potential recognition decision.

\section{Determinant value}

The term determinant of a square matrix $\mathrm{A}$, denoted $\operatorname{det}(\mathrm{A})$ or $|\mathrm{A}|$, refers to both the collection of the elements of the square matrix, enclosed in vertical lines, and the scalar value represented by that array. Thus,

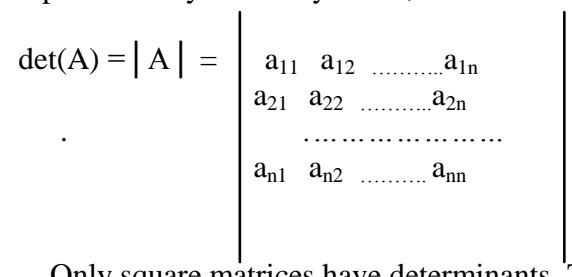

Only square matrices have determinants. The scalar value of the determinant of a $2 \times 2$ matrix is the product of the elements on the major diagonal minus the product of the elements on the minor diagonal. Thus,

$$
\begin{aligned}
& \operatorname{det}(A)=|A|=\left|\begin{array}{cc}
a_{11} & a_{12} \\
a_{21} & a_{22} \\
&
\end{array}\right| \\
& =a_{11} a_{22} a_{12} a_{21} \quad \ldots \ldots \ldots \ldots \ldots \ldots \ldots \ldots \ldots \ldots \ldots
\end{aligned}
$$

The scalar value of the determinant $\mathrm{f}$ a $3 \times 3$ matrix is composed $\mathrm{f}$ the sum $\mathrm{f}$ six triple products which can be obtained from the augmented determinant [6]:

$$
\operatorname{det}(A)=|A|=\left|\begin{array}{ccccc}
a_{11} & a_{12} & a_{13} & a_{11} & a_{12} \\
a_{21} & a_{22} & a_{23} & a_{21} & a_{22} \\
a_{31} & a_{32} & a_{33} & a_{31} & a_{32}
\end{array}\right|
$$

The $3 \times 3$ determinant is augmented by repeating the first two columns $f$ the determinant on the right-hand side of the determinant. Three triple products are formed, starting with the elements of the first row multiplied by the two remaining elements on the downward-sloping diagonals. Three more triple products are formed, starting with the elements of the third row multiplied by the two remaining elements on the right-upward sloping diagonals. The value of the determinant is the sum of the first three triple products minus the sum of the last three triple products. Thus [6],

$\operatorname{det}(\mathrm{A})=|\mathrm{A}|=a_{11} a_{22} a_{33}+a_{12} a_{23} a_{31}+a_{13} a_{21} a_{32}--a_{31} a_{22} a_{13^{--}}$ $a_{32} a_{23} a_{l l}-a_{33} a_{21} a_{12} \ldots \ldots \ldots \ldots \ldots \ldots$.

(4)

\section{The Recognition Methodology}

Suppose you have $\mathrm{M}$ images of the training set are submitted to a foot. All images must be in same size (e.g. $n \times n$ pixels).

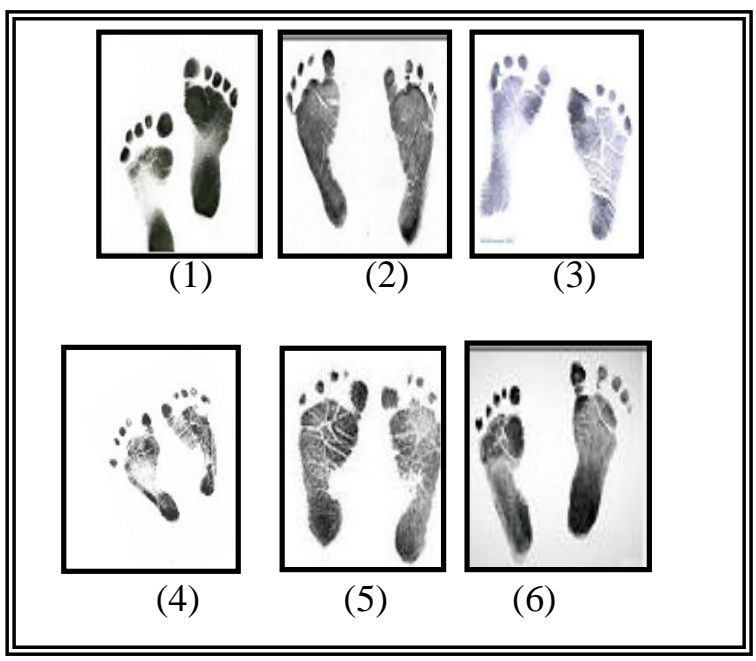

Fig 1: Training set of six foot images, each of $256 \times 256$ pixels size

Each image subdivided in to blocks $C_{k}$ the size of each blocks are $3 \times 3$ then find determinant value $\operatorname{det}(\mathrm{A})$ for each blocks which illustrated in Eq.2

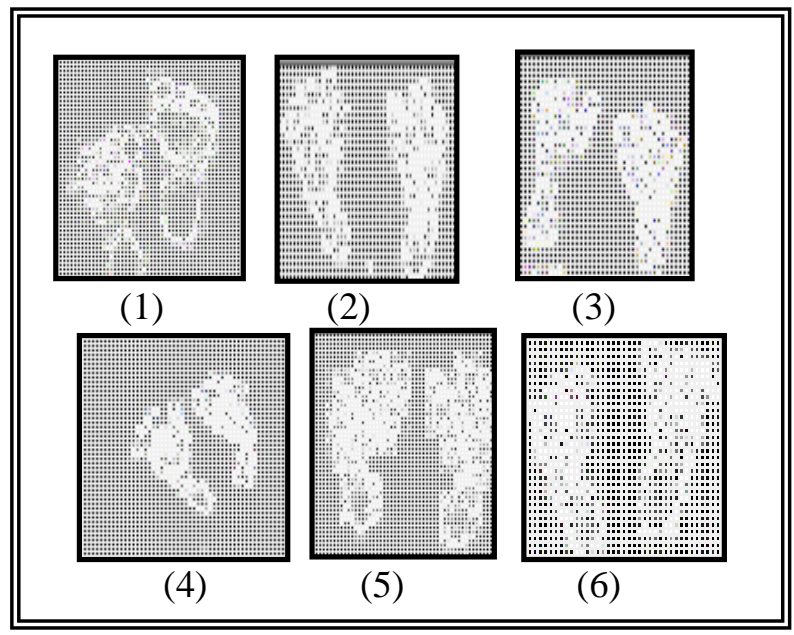

Fig 2: Determinant value for each image using $(3 \times 3)$ blocks

A threshold, T, is used to convert the image to black and extract features, $\mathrm{I}(\mathrm{x}, \mathrm{y})$, as shown in

$$
\begin{gathered}
\text { IF } \quad \text { I(i,j) } \geq T \rightarrow I(i, j) \\
\text { Otherwise } \\
I(i, j) \rightarrow 0
\end{gathered}
$$




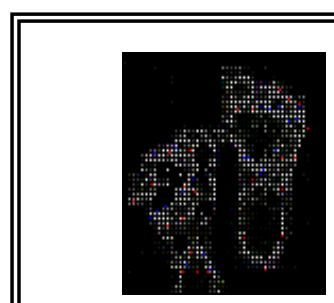

(1)

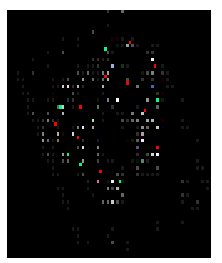

(4)

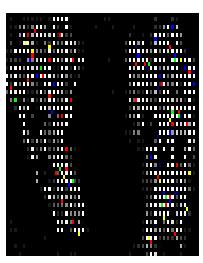

(2)

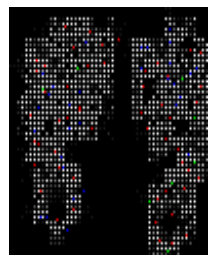

(5)

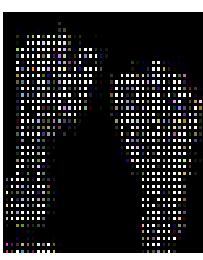

(3)

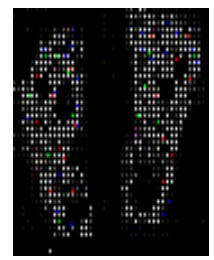

(6)
Fig 3: Extract Features for each image using threshold (T)

The next stage in our approach is determining centric point. The information we have up to now is the boundary of foot as sequential coordinates in the image. Now, we show that using this boundary pixels and centric point of boundary image Centric point of the foot boundary image which is calculated by the equations $(5,6,7)$ Here $f(i, j)$ is

our hand image function and $X_{\text {Centriod }} Y_{\text {Centriod }}$ is the coordinates of the centric point.

$$
m_{p q}=\sum_{i=-\infty}^{\infty} \sum_{j=-\infty}^{\infty} i^{p} j^{q} f(i, j)
$$

$$
\begin{aligned}
& X_{\text {Centroid }}=\frac{m_{1 \mathrm{O}}}{m_{\mathrm{OO}}} \\
& Y_{\text {Centroid }}=\frac{m_{\mathrm{O} 1}}{m_{\mathrm{OO}}}
\end{aligned}
$$

We calculate the Euclidean distance between each pixel (x, y) on the boundary and the centric point as equation (8) shown in Table-1.

$$
D(x, y)=\sqrt{\left(x-x_{\text {centriod }}\right)^{2}+\left(y-y_{\text {centriod }}\right)^{2}}
$$

Using these key points, we find points $\mathrm{X}_{1}, \mathrm{X}_{2}, \mathrm{X}_{3}, \mathrm{X}_{4}$ that are the initial points and end points of the holes between fingers of foot in the boundary image. A sample of the way we find these points is shown in Fig.3. We find $H_{1}$ (centric point) and then calculate Euclidean distance of pixels on the boundary.
Finally ,Recognition for partially occluded and overlapping objects in composite scenes. The objects are matched against the scene and their position is recovered. Not effectively applicable to rotation, translation and scale invariant cases. Rate of recognition is Successful[7]. As it is obvious, the similarity between the verify feet and the trained set can be represented by the minimum distance test (i.e. utilizing the Mean-Square-Error "MSE" criterion), given by:

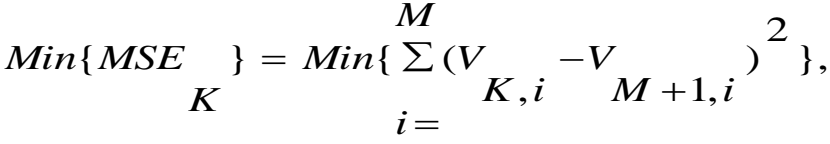

$$
\begin{aligned}
& \text { for } K=1,2, \ldots, M
\end{aligned}
$$

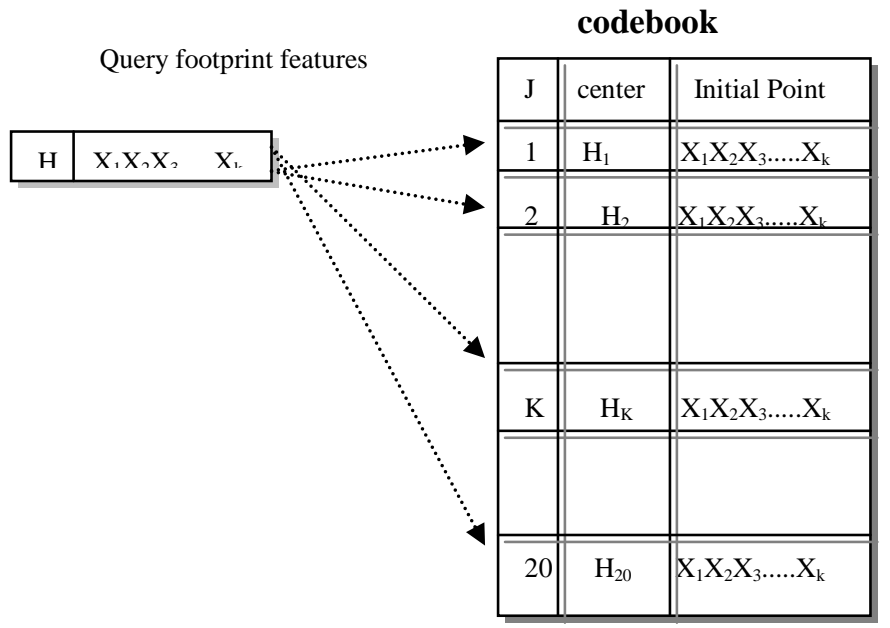

Fig 4: The training set of foot images plus an existed test foot to be verified

Table1.Euclidian Distance between initial point and centric point for each image

\begin{tabular}{||c|c|c|c|c|c|c||}
\hline Item & $\begin{array}{c}\text { ED } \\
\text { Foot }_{\mathbf{1}}\end{array}$ & $\begin{array}{c}\text { ED } \\
\text { Foot }_{\mathbf{2}}\end{array}$ & $\begin{array}{c}\text { ED } \\
\text { Foot }_{\mathbf{3}}\end{array}$ & $\begin{array}{c}\text { ED } \\
\text { Foot }_{\mathbf{4}}\end{array}$ & $\begin{array}{c}\text { ED } \\
\text { Foot }_{\mathbf{5}}\end{array}$ & $\begin{array}{c}\text { ED } \\
\text { Foot }_{\mathbf{~}}\end{array}$ \\
\hline \hline $\mathbf{1}$ & 3.021 & 1.33 & 6.112 & 12.9 & 1.88 & 5.139 \\
\hline $\mathbf{2}$ & 1.202 & 8.11 & 2.118 & 3.226 & 4.114 & 5.222 \\
\hline $\mathbf{3}$ & 2.901 & 2.591 & 1.15 & 3.25 & 7.742 & 8.995 \\
\hline $\mathbf{4}$ & 3.55 & 2.86 & 2.17 & 1.48 & 0.79 & 0.099 \\
\hline $\mathbf{5}$ & 1.221 & 3.453 & 8.123 & 6.811 & 2.345 & 7.603 \\
\hline \hline $\mathbf{6}$ & 3.021 & 1.33 & 6.112 & 1.921 & 2.324 & 5.139 \\
\hline
\end{tabular}

\section{EXPERIMENT AND EVALUATION}

To test the general applicability of the proposed approach, the Footprint recognition system was built to distinguish the query footprint in between dataset. The camera takes the image. This research shows that the shapes of pattern spectra differ from men \& women. Widely used in aerial defense, outer space exploration, medicine, etc.. Features of footprint image are 
defined from Angular relation, Length, Region. In the following an explanation about input/output details and the evaluation of the proposed system:

\subsection{Input FootPrint Dataset}

The footprint dataset are recorded by Scanner under windows operating system with an attributes 24-BMP image. We used the database for footprints recognition experiments. Here we have experimented with nearly 60 images with variations of 40 persons taken by 8 sample; male, female, and children.

\subsection{Output Results}

. The experiment results show the effectiveness of the proposed method. They show clearly the flexibility of the method in relation step translation of the footprints. A preview image of the Database of foot is as shown in Fig- $(4,5)$.The experimental observations of the experiments performed on datasets are shown in Table- 2 and Table- 3 as follows:

\subsection{Evaluation}

In the current computer implementation of determinant problem based class and mapping, a careful computation treatment was found needed especially with that related to

the variables precision and the normalization of determinant values. Thereby, the numerical experiments indicate quite optimistic availability of the proposed algorithm for footprints recognition system .Suppose an existed image within the trained set has been selected to be verified, as illustrated in Fig.4.

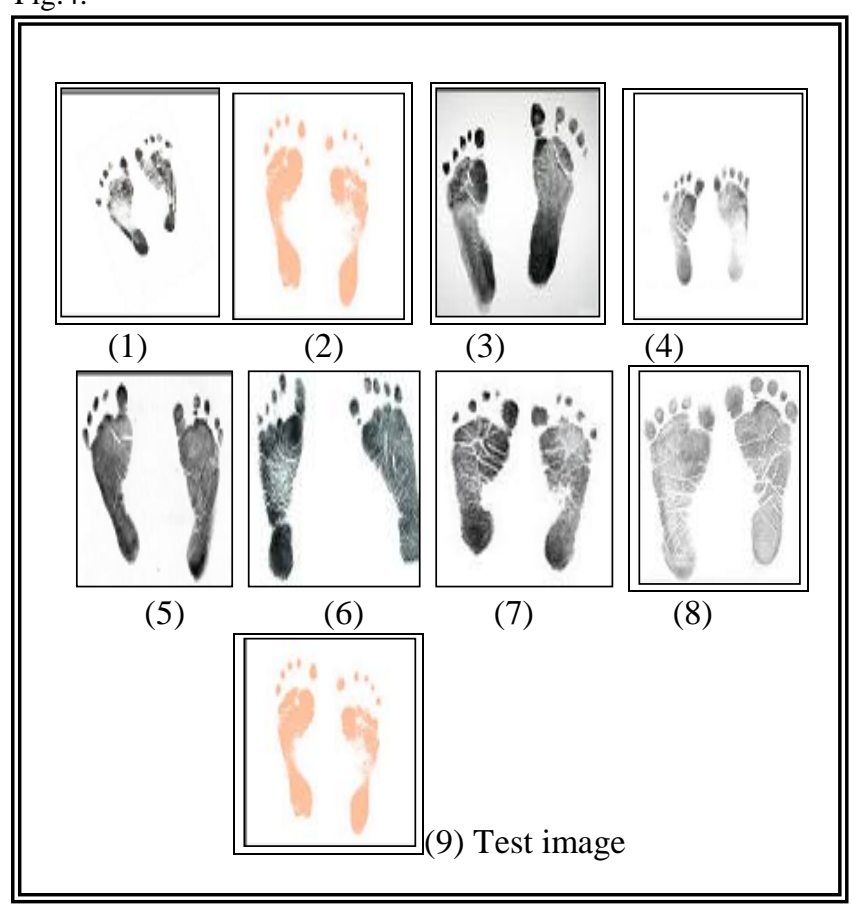

Fig 5:The training set of foot images plus an existed test foot image to be verified

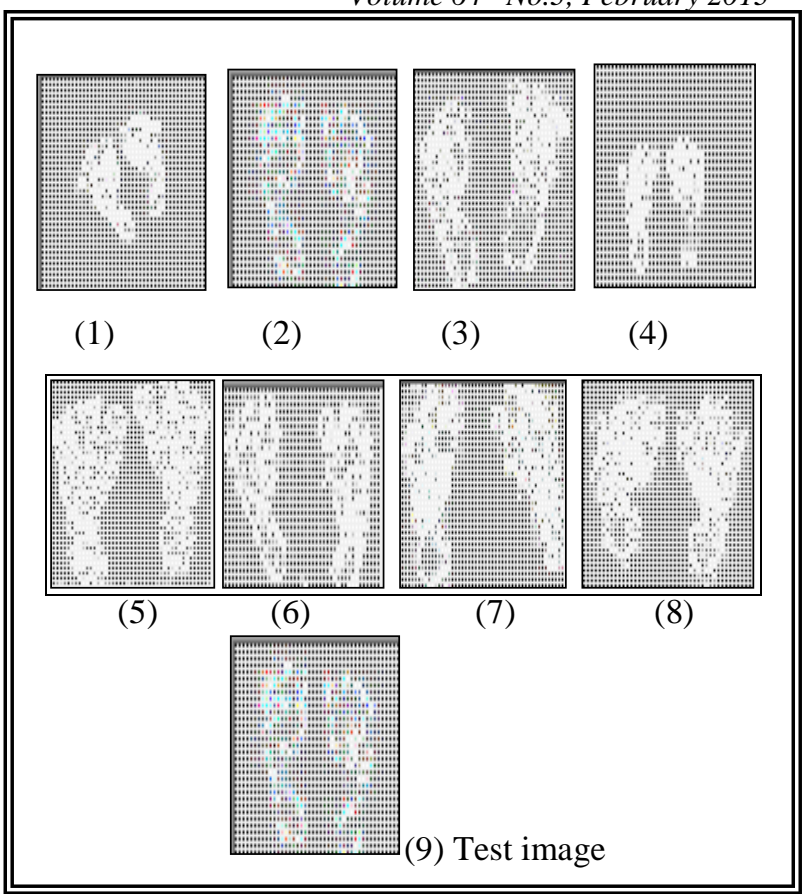

Fig 6: The training set of foot images plus an existed test foot to be verified when we find determinant value each block

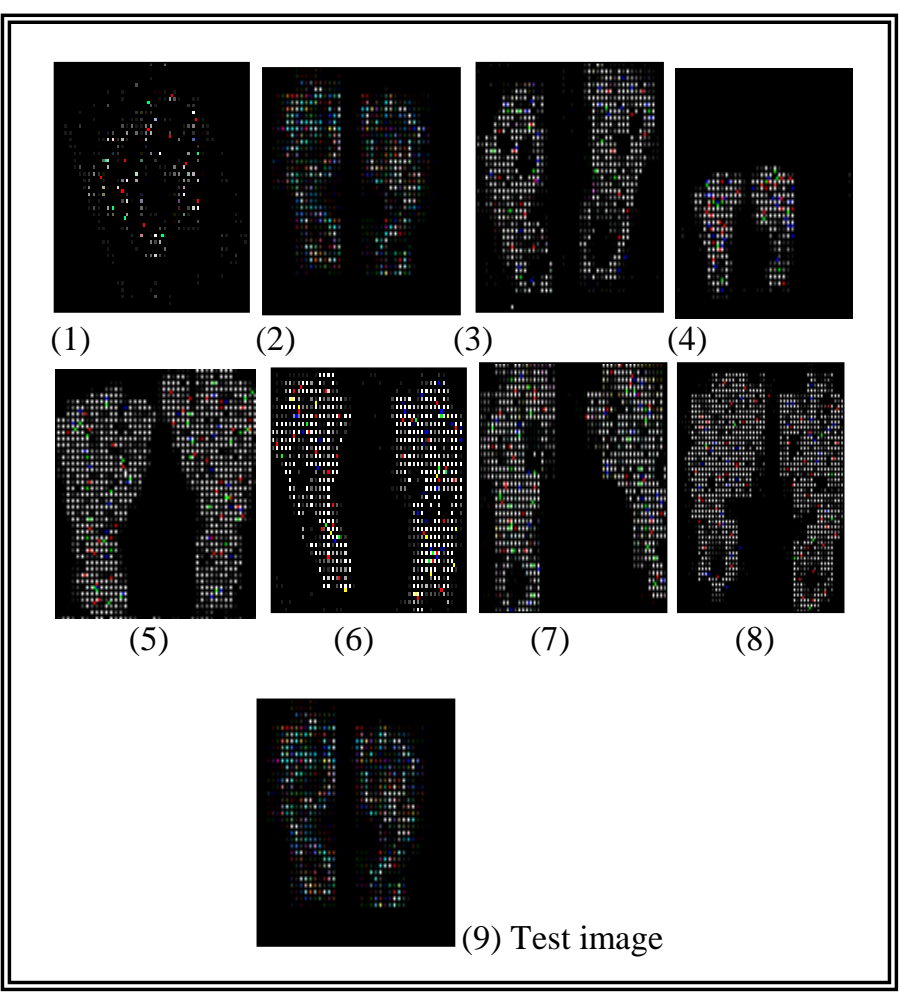

Fig 7: Extract Features for each image using Threshold (T)

The Euclidean Distance between trained set and the verifying Foot image is, obvious as listed below: 
Table 2. Euclidian Distance between initial point and centric point for each image and verified image

\begin{tabular}{|c|c|c|c|c|c|c|c|c|c|}
\hline \multirow{2}{*}{ len } & ED & ED & ED & ED & ED & ED & ED & ED & ED \\
\hline & Foot $_{1}$ & $\mathrm{Foot}_{2}$ & $\mathrm{Foot}_{3}$ & $\mathrm{Foot}_{4}$ & Foot $_{5}$ & Foot $_{\sigma}$ & $\mathrm{Foot}_{7}$ & $\mathrm{Foot}_{8}$ & Footg \\
\hline 1 & 13,221 & 21115 & 11.009 & 10.097 & 1.203 & 2.309 & 3.415 & 4.521 & 2.115 \\
\hline 2 & 2.202 & 4.11 & 6.018 & 7.926 & 9.834 & 11.742 & 13.65 & 15.558 & 3.11 \\
\hline 3 & 11,922 & 21111 & 2.3 & 122489 & 2.6781 & 2.867 & 8.356 & 3.245 & 0.111 \\
\hline 4 & 2.1511 & 2321 & 2.4911 & 12.661 & 2.831 & 12.001 & 3.171 & 173231 & 2321 \\
\hline 5 & 9.221 & $5,4.13$ & 5.605 & 15.213 & 5.989 & 6.181 & 6.373 & 6.565 & 5.413 \\
\hline 6 & 14,121 & 3323 & 2.525 & 1.727 & 0.929 & 10.131 & 0.6671 & 1.465 & 33213 \\
\hline 7 & 9.2311 & 5921 & 2.611 & 0.699 & 4,0091 & 173.319 & 10.629 & 13939 & 5.212 \\
\hline 8 & 16.219 & 6885 & 7.5511 & 8.217 & 8.883 & 9.549 & 10.215 & 108881 & 6.534 \\
\hline
\end{tabular}

The Min $\{M S E\}$ between trained set and the verifying Foot image is, obvious, between ED Foot2 and ED Foot9 i.e. Min $\left\{M S E_{K}\right\}=M S E_{2}$, which illustrated in $\boldsymbol{E q}$.(9) as listed below:

\begin{tabular}{|c|c|c|c|c|c|c|c|c|}
\hline $\mathrm{NO}$ & $\mathrm{MSE}_{1}$ & $\mathrm{MSE}_{2}$ & $\mathrm{MSE}_{3}$ & $\mathrm{MSE}_{4}$ & $\mathrm{MSE}_{5}$ & $\mathrm{MSE}_{6}$ & $\mathrm{MSE}_{7}$ & $\mathrm{MSE}_{8}$ \\
\hline 1 & 11.106 & 0 & 8.894 & 7.982 & 0.912 & 0.194 & 1.3 & 2.406 \\
\hline 2 & 0.908 & 1 & 2.908 & 4.816 & 6.724 & 8.632 & 10.54 & 12.448 \\
\hline 3 & 11.811 & 2 & 2.189 & 12.378 & 2.5671 & 2.756 & 8.245 & 3.134 \\
\hline 4 & 0.1699 & 0 & 0.1701 & 10.34 & 0.51 & 9.68 & 0.85 & 15 \\
\hline 5 & 3.808 & 0 & 0.192 & 9.8 & 0.576 & 0.768 & 0.96 & 1.152 \\
\hline 6 & 10.798 & 0 & 0.798 & 1.596 & 2.394 & 6.808 & 2.6559 & 1.858 \\
\hline 7 & 4.0191 & 0.709 & 2.601 & 4.513 & 1.2029 & 12.107 & 5.417 & 8.727 \\
\hline 8 & 9.685 & 0.351 & 1.0171 & 1.683 & 2.349 & 3.015 & 3.681 & 4.347 \\
\hline
\end{tabular}

Table 3. Mean square Error between training set of Foot image and verify image

\section{Conclusion}

In this paper, a complete and fully automatic approach for Human footprint recognition images is proposed. The algorithms used in footprint recognition are categorized into three stages: image gathering processing, feature extraction and template matching .our recognition make approach computationally inexpensive and significantly robust recognition decision Using three-stage feature matching with centric point and boundary edge of footprint measures significantly improved the matching performance. Comparative with other methods a high recognition rate by verifying raw footprints directly is difficult to obtain, because people stand in various positions with different distances and angles between the two feet. To achieve robustness in matching an input pair of Footprints with those of registered footprints, the input pair of footprints must be normalized in position and direction. Such normalization might remove useful information for recognition.

\section{ACKNOWLEDGMENT}

I'd like to thank all those people who helped during the development of this manuscript, by reviewing parts of the manuscript, by general feedback, or by other assistance. Those people include Abbas AL-phalahi, Aiman Abudul Razzak, and IJCA Editors. I also received valuable contributions from those anonymous reviewers of an early draft whose favorable opinions were vital to getting this manuscript published. To all of these people: your comments and suggestions have helped make this manuscript considerably better than it might have been. To the anonymous reviewers: thanks for the favorable opinions!

\section{REFERENCES}

[1] S. Liu and M. Silverman, "A practical guide to biometric security technology", IEEE IT Pro, pp.27-32, Jan. /Feb., 2001.

[2] L. C. Jain et al., Ed., Intelligent Biometric Techniques in Fingerprint and Face Recognition. Boca Raton, FL: CRC Press, 1999.

[3] V.D. Ambeth Kumar , Chennai, India," Legacy of Footprints Recognition- A Review" International Journal of Computer Applications (0975 - 8887) Volume 35- No.11, December 20119

[4] V. D. Ambeth Kumar1 M. "Footprint Recognition using Modified Sequential Haar Energy Transform (MSHET)", Ramakrishnan Research scholar in sathyabama university, Chennai, Tamil Nadu- 600 119, India. Professor and Head of Department Of Information Technologies, Velammal Engineering College, Chennai, Tamil Nadu.

[5] Young W. Woo "Performance Evaluation of Binarizations of Scanned Insect Footprints", Division of Computer \& Visual Engineering College of Engineering, Dongeui University San 24, Gaya-Dong, Pusanjin-Gu, Pusan, 614714 , Korea

[6] Joe D. Hoffman ,"Numerical Methods for Engineers and Scientists"Second Edition Revised and Expanded Department of Mechanical Engineering Purdue University West Lafayette, Indiana MARCEL DEKKER Copyright () 2001 by Marcel Dekker, Inc. All Rights Reserved.

[7] J.Hong and H. J. Wolfson, "An Improved Model-Based Matching Method Using Footprints," Robotics Report 137, Computer Science Div., Courant Inst. of Math., N W ,1987

[8] R. R. Coifman and L. Donoho, "Translation Invariant Denoising,” Dept. Statist., Stanford Univ., Tech. Rep. 475, 1995. 
[9] K. Ramchandram and M. Vetterli, "Best wavelet packet bases in a ratedistortion sense," IEEE Trans. Image Processing, vol. 2, pp. 160-175, Apr. 1993.

[10] D. L. Donoho and P. B. Stark, "Uncertainty principles and signal recovery," SIAM J. Appl. Math., vol. 49/3, pp. 906931, June 1989.

[11] M. Elad and A. M. Bruckstein, "On sparse signal representations," in IEEE Int. Conf. Image Processing, Thessaloniki, Greece, Oct. 2001.

[12] M. M. Goodwin and M. Vetterli, "Matching pursuit and atomic signal models based on recursive filter banks," IEEE Trans. Signal Processing, vol. 47, pp. 1890-1902, July 1999.

[13] V.D.Ambeth Kumar and M.Ramakrishan ,'Footprint Recognition using Modified Sequential Haar Energy Transform (MSHET)", Intl. Journal of Computer science Issuse,Vol-7,Issuse 3,No.5,May 2010.
[14] Jain AK, Ross A, Prabhakar S (2004) An introduction to biometric recognition. IEEE Trans Circuits Syst Video Technol Special Issue Image Video based Biometrics 14(1):4-20

[15] Zhang D, Kong A, You J, Wong M (2003) Online palmprint identification.IEEE Trans Pattern Anal Mach Intell 25(9):1041-1050

[16] Zhang D, Guo ZH, Lu GM, Zhang L, Zuo WM (2010) An online system of multispectral palmprint verification. IEEE Trans Instrum Meas 59(2):480-490

[17] K. Nakajima, Y. Mizukami, K. Tanaka, and T. Tamura, "Foot-Based Personal Recognition", IEEE: Tr. On Biomedical Engineering, Vol. 47, No. 11, 2000.

[18] Dynamic-Footprint based Person Identification using Mattype Pressure Sensor in-Woo Jungl, Zeungnam Bien', Sang-Wan Lee', Tomomasa Sato', IEEE 2003. 